\title{
Severe haemoptysis in patients with nonsmall cell lung carcinoma
}

\author{
Keyvan Razazi ${ }^{1}$, Antoine Parrot ${ }^{1}$, Antoine Khalit ${ }^{2}$, Michel Djibre ${ }^{1}$, \\ Valerie Gounant ${ }^{3,4}$, Jalal Assouad ${ }^{4,5}$, Marie France Carette ${ }^{2,5}$, \\ Muriel Fartoukh ${ }^{1,5}$ and Jacques Cadranel ${ }^{3,5}$
}

\begin{abstract}
Affiliations: ${ }^{1}$ AP-HP, Hôpital Tenon, Unité de Réanimation Médico-Chirurgicale, Pôle Thorax Voies Aériennes, Groupe Hospitalier des Hôpitaux Universitaires de l'Est Parisien, Paris, France. ${ }^{2}$ AP-HP, Hôpital Tenon, Service de Radiologie, Pôle Imagerie, Groupe Hospitalier des Hôpitaux Universitaires de l'Est Parisien, Paris, France. ${ }^{3}$ AP-HP, Hôpital Tenon, Service de Pneumologie - Centre Expert en Oncologie Thoracique, Pôle Thorax Voies Aériennes, Groupe Hospitalier des Hôpitaux Universitaires de l'Est Parisien, Paris, France. ${ }^{4}$ AP-HP, Hôpital Tenon, Service de Chirurgie Thoracique, Pôle Thorax Voies Aériennes, Groupe Hospitalier des Hôpitaux Universitaires de l'Est Parisien, Paris, France. ${ }^{5}$ Sorbonne Universités, UPMC Univ Paris 06, Paris, France.
\end{abstract}

Correspondence: Antoine Parrot, AP-HP, Hôpital Tenon, Unité de Réanimation Médico-Chirurgicale, Service de Pneumologie - Centre Expert en Oncologie Thoracique, Pôle Thorax Voies Aériennes, Groupe Hospitalier des Hôpitaux Universitaires de l'Est Parisien, F-75970 Paris Cedex 20, France.

E-mail: antoine.parrot@tnn.aphp.fr

ABSTRACT Severe haemoptysis due to nonsmall cell lung cancer (NSCLC) is considered a grim condition, and there is still scarce data on its characteristics and outcome, despite new imaging and treatment modalities. This retrospective study sought to describe the clinical characteristics, pathophysiology and outcome of NSCLC-related haemoptysis.

We included 125 consecutive patients with severe haemoptysis (>100 mL) at admission, 65 (52\%) exhibiting squamous cell carcinoma. Tumour cavitation/necrosis was reported in 26 (21\%) patients. 52 patients had received anticancer treatment, but none had received anti-angiogenic agents.

Severe haemoptysis was related mainly to the bronchial artery (82\%), and major pulmonary artery involvement was rare (6.4\%). Interventional radiology was performed in 102 patients. Bleeding cessation was achieved in 108 (87\%) out of 125 patients. The overall in-hospital and 1-year survival rates were 69\% and $30 \%$, respectively. Performance status (PS) $\geqslant 2$ (OR 3.6, 95\% CI 1.3-9.6), advanced stage (OR 8.6, 95\% CI 2-37) and mechanical ventilation (OR 13, 95\% CI 4.5-36) were independent predictors of in-hospital mortality. Performance status $\geqslant 2$ (hazard ratio (HR) 2.4, 95\% CI 1.5-3.7), advanced stage (HR 4, 95\% CI 2.1-7.7), cancer progression (HR 2, 95\% CI 1.01-2.7) and cavitation/necrosis (HR 1.7, 95\% CI 1.21-3.2) were independently associated with 1 -year mortality.

Management of severe haemoptysis related to NSCLC should be improved, given our observed survival rates after hospital discharge.

@ERSpublications

NSCLC-related haemoptysis should not be considered dismissively; its management is still suboptimal http://ow.ly/CeI3S

For editorial comments see Eur Respir J 2015; 45: 601-603 [DOI: 10.1183/09031936.00199914].

Received: Jan 132014 | Accepted after revision: Sept 222014 | First published online: Oct 302014

Conflict of interest: None declared.

Copyright OERS 2015 


\section{Introduction}

During the clinical course of nonsmall cell lung cancer (NSCLC), 20-60\% of patients will develop haemoptysis, with $5-10 \%$ of cases considered severe. Without treatment, severe haemoptysis is associated with a mortality rate exceeding 50\% [1-4]. Recently, this serious complication was reported in six $(9.1 \%)$ out of 66 bevacizumab-treated patients, with a fatal outcome in four (67\%) [5]. Since the 1990s, no new data has been published concerning the clinical characteristics, pathophysiology and optimal management of cancer-related severe haemoptysis [6,7]. Interventional radiology is considered first-line treatment for severe haemoptysis, while the American College of Chest Physicians (ACCP) guidelines currently recommend bronchoscopic techniques to control haemoptysis in this setting [3]. The lack of data may be partly explained by physicians' unwillingness to treat cancer-related severe haemoptysis, as they consider the procedures, especially bronchial arteriography embolisation (BAE), futile. More data is, therefore, required on optimal management and its impact on short- and long-term survival [8]. We describe the clinical characteristics, pathophysiology and management options, as well as in-hospital and 1-year survival rates, in consecutive patients with NSCLC-related severe haemoptysis.

\section{Methods}

The study was conducted between May 1, 1995 and January 1, 2010 in a tertiary teaching hospital and referral centre for lung cancer and haemoptysis in Paris, France. All consecutive patients admitted to the respiratory intermediate care ward or intensive care unit (ICU) with haemoptysis related to histologically-proven NSCLC were eligible. Exclusion criteria were bleeding episodes related to causes other than cancer, such as bronchiectasis or pulmonary embolism, or bronchial biopsy-induced trauma. For patients with recurrent haemoptysis only the first episode was considered.

\section{Patient characteristics}

For each patient, the following information was retrieved from a prospective database. 1) Epidemiological and clinical data at admission, such as age, sex, smoking status, alcohol consumption, preadmission Eastern Cooperative Oncology Group performance status (PS) score assessed within 1 week prior to ICU admission [9, 10], comorbidities, and ongoing anticoagulant or antiplatelet treatment. 2) Cancer characteristics, such as histological type using 2004 World Health Organization pathological classification, extent using TNM (tumour, node, metastasis) classification [11], and prior and current specific treatments (surgery, chemotherapy, anti-angiogenic agents, epidermal growth factor tyrosine kinase inhibitors (e.g. erlotinib or gefitinib) or radiation therapy). Advanced disease was defined as stages IIIB or IV. Cancer status was defined as controlled (response plus stable disease), progressive or unknown. Unknown status comprised patients waiting for a decision to treat, receiving ongoing first-line chemotherapy, or diagnosed with lung cancer during the ICU stay. Tumour location (central or peripheral) and presence of cavitation or necrosis were evaluated using fibre-optic or radiology findings [12, 13].

\section{Haemoptysis characteristics and management}

The following criteria have been used in our centre over the past 15 years to define severe haemoptysis: 1) cumulative expectorated amount $>100 \mathrm{~mL}$ within 1 week; 2) respiratory failure requiring high-flow oxygen or mechanical ventilation; 3) haemodynamic instability; 4) severe comorbidities (e.g. chronic pulmonary disease) potentially causing life-threatening haemoptysis; and 5) requirement for vasoconstrictive drugs. Fatal haemoptysis was defined as cardiac arrest or death related to severe haemoptysis. Acute illness severity was assessed using the Simplified Acute Physiology Score (SAPS) II [14].

We attempted to elucidate haemoptysis mechanism using pathological data if available, and multidetector CT-angiography (MDCTA) [15] or interventional radiology data including pulmonary angiography if performed. If bleeding stopped following BAE, bronchial artery involvement was considered the main mechanism of haemoptysis.

Regarding management, all invasive and noninvasive therapeutic interventions during hospital stay were recorded, such as bronchoscopy, bronchial arteries embolisation, pulmonary endovascular occlusion or stenting, systemic vasoconstrictive agents, or surgery. Haemoptysis recurrence was followed up at 3 months.

\section{Survival}

Survival was evaluated using in-ICU, in-hospital and 1-year rates. Vital status was to be recorded at 12 months. Specific anticancer treatments after ICU discharge, such as chemotherapy, radiation therapy or surgery, were collected. 


\section{Statistical analysis}

Data are expressed as median (interquartile range) or $\mathrm{n}(\%)$. Quantitative and nominal variables were compared using the unpaired t-test or the Mann-Whitney U-test, and the Chi-squared test or Fisher's exact test, as appropriate.

Prognostic factors were studied in the entire cohort, with univariable analysis used first to assess the association between each variable and mortality. The variables selected by univariable analysis $(\mathrm{p}<0.15)$ as well as the events considered clinically relevant were entered into a logistic regression model with respect to the ratio of one variable per 10 events [16]. SAPS II data was not included in multivariable analysis, as other variables like age and mechanical ventilation, were already included. Results were expressed as crude and adjusted odds ratios, with $95 \%$ confidence intervals. Model discrimination was assessed using the Hosmer-Lemeshow test.

Overall survival following severe haemoptysis was defined as the time from date of ICU entry to the time of death from any cause or the last follow-up visit, in the whole population and in those discharged from hospital. Survival analysis was censored at 12 months, a length of time we consider appropriate for assessing haemoptysis management in lung cancer. Univariable Cox proportional hazards regression was used to evaluate predictors of 1 -year mortality. Only those variables that were significantly associated with 1 -year mortality $(p<0.15)$ in the univariable analysis were entered into the multivariable model. Results were reported as hazard ratios and $95 \%$ confidence intervals. Statistical analyses were performed using Stata software, Version 10.1 (StataCorp LP, College Station, TX, USA).

The study was conducted in accordance with the French National Commission for Protection of Patients' Rights and Electronic Data Recording (www.cnil.fr) and French regulatory requirements. Due to the retrospective study design, neither institutional review board approval nor patient written informed consent were required.

\section{Results}

\section{Patient characteristics}

A patient flow chart is provided in figure 1 and patient characteristics in table 1 . The majority of patients were male (111 (89\%) out of 125$)$, with a median age of $62(53-71)$ years, and one-third had a PS $\geqslant 2$. The histological cancer type was squamous cell carcinoma (SCC) in 65 (52\%) patients, adenocarcinoma in 31 (25\%), and other in 29 (23\%) (large cell carcinoma $\mathrm{n}=21$; "not otherwise specified NSCLC" $\mathrm{n}=6$; and pleomorphic carcinoma $n=2$ ). Overall, 87 patients exhibited stage IIIB or IV NSCLC. None had received anti-angiogenesis treatment. Tumour necrosis or cavitation was reported in 26 (21\%) patients and was mainly observed in SCC patients (16 (62\%) out of 26).

\section{Haemoptysis characteristics}

Median expectorated haemoptysis volume at admission was 200 (100-300) mL. Median SAPS II score was 27 (31-38) points. Mechanical ventilation was required in 38 (30\%) patients, either within $24 \mathrm{~h}$ of ICU admission $(n=33)$ or during ICU stay $(n=5)$.

Haemoptysis was definitely related to cancer extent with pulmonary artery erosion in eight (6.4\%) patients and systemic arterial rupture in two (2\%) patients. Otherwise, haemoptysis was secondary to bleeding within the tumour. Bleeding was, thus, definitely related to bronchial artery involvement in $65(52 \%)$ patients. In 37 (30\%) patients, severe haemoptysis was possibly related to bronchial artery involvement (normal MDCTA, normal pulmonary angiography or BAE failure). In the remaining 13 (10\%) patients, the exact mechanism could not be established.

Fatal haemoptysis occurred in 22 (18\%) patients, eight (36\%) and 10 (45\%) of whom exhibited SCC and adenocarcinoma, respectively, with no link found between histological type and fatal haemoptysis $(\mathrm{p}=0.07)$. Although not statistically different, the proportion of cavitation (7 (32\%) out of 22 versus 19 (18\%) out of 103; $\mathrm{p}=0.25)$ and central location $(19(90 \%)$ out of 21 versus 72 (72\%) out of $101 ; \mathrm{p}=0.09)$ was higher in patients with fatal compared with those with nonfatal haemoptysis. The mechanism was thought to be related to bronchial artery involvement $(n=14)$ or major vessel involvement $(n=2)$, or was unclear $(n=6)$.

To control active bleeding, all patients received conservative measures as described elsewhere [17], including systemic terlipressin in 57 (46\%) and bronchoscopy in 34 (27\%) patients (36 procedures), combining blood aspiration, local instillation of cold saline lavage $(n=21)$ and local vasoconstrictor administration $(n=29)$. In one case, balloon bronchial tamponade was employed. Conservative procedures were the sole treatment given in 20 (16\%) patients for various causes (data not shown), with haemoptysis control achieved in 14. In the six remaining patients, BAE could not be performed in time due to fatal haemoptysis $(n=3)$, physical disabilities (bedridden patients: $n=2)$ or technical issues $(n=1)$. 


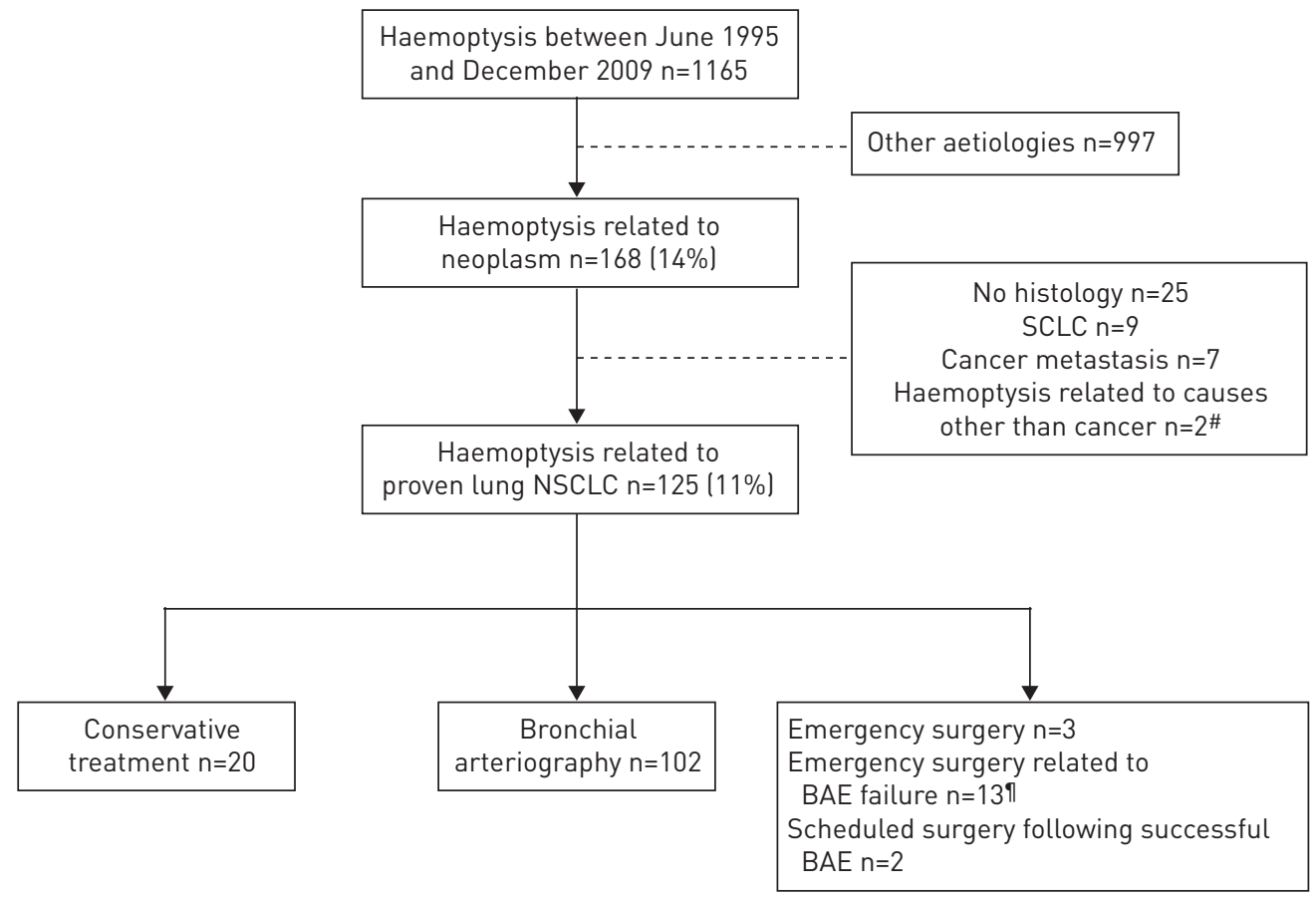

FIGURE 1 Flow chart of patients admitted for severe haemoptysis to Tenon Hospital (Paris, France) between June, 1995 and December, 2009. NSCLC: nonsmall cell lung cancer; SCLC: small cell lung cancer; BAE: bronchial arteriography embolisation. " : bronchiectasis $\mathrm{n}=1$ and pulmonary embolism $\mathrm{n}=1$; ${ }^{\text {ๆ: }}$ emergency surgery in patients in whom bleeding was not controlled after BAE.

Regarding invasive procedures, five patients underwent pulmonary endovascular procedures (stent graft in two patients and transcatheter coil embolisation in the three others); 122 bronchial arteriography procedures were performed in 102 patients, and completed with bronchial embolisation of affected arteries in 89 cases. No severe procedure-related complications occurred. Of the 102 patients, bleeding cessation was achieved in $82(80 \%)$. Surgery was performed in 18 patients: emergency surgery alone on account of potential pulmonary artery involvement in three patients, pulmonary artery occlusion being considered technically unfeasible; emergency surgery following BAE failure in 13 patients; and curative oncological surgery in the remaining two.

In the entire cohort, bleeding cessation was achieved in 108 (87\%) out of 125 patients, irrespective of the treatment type, and bleeding recurrence within 3 months was observed in 20 (16\%) patients.

\section{Survival}

Vital status was obtained at 1 year for all but two patients. ICU, in-hospital, and 1-year survival rates were $83 \%, 69 \%$, and $30 \%$, respectively. Median survival after haemoptysis was $4.4(0.6-16)$ months in the entire study cohort, and 9.9 (4-20) months among the 86 (69\%) patients alive on hospital discharge. In the patients treated using either conservative measures or interventional radiology, in-hospital survival rates were $10(50 \%)$ out of 20 patients and $68(67 \%)$ out of 102, respectively. Of the 38 patients under mechanical ventilation, 30 underwent bronchial arteriography and three surgery. Their median survival was poor (0.5 (0.1-3.7) months), and only 13 patients from this subset were discharged from hospital. Of the 39 patients who died during hospital stay, causes of death were haemoptysis $(n=22)$, cancer progression $(n=11)$, infection $(n=3)$, pulmonary embolism $(n=1)$, cardiac arrhythmia $(n=1)$ and acute renal failure $(\mathrm{n}=1)$.

PS $\geqslant 2$, advanced stage and mechanical ventilation were independent predictors of in-hospital mortality (table 2). The final model showed good calibration and discrimination (Hosmer-Lemeshow goodness-of-fit $\mathrm{p}=0.78$, Chi-squared $=1.78$; area under the receiver operating characteristic curve $=0.85$ ). Advanced stage, PS $\geqslant 2$, cancer progression, cavitation/necrosis, as well as non-SCC, were independently associated with 1-year mortality (table 3 ).

Of note, $60(48 \%)$ patients, 15 of whom required mechanical ventilation, received anticancer treatment following ICU discharge, such as surgery $(n=12)$, radiation therapy $(n=12)$ or chemotherapy $(n=51)$. 


\section{TABLE 1 Patient characteristics}

\section{Factors at ICU admission}

Age years

$62(53-71)$

Alcohol abuse

$31(24.8)$

Performance status

0

$32(25.6)$

1

$47(37.6)$

$35(28)$

3

$10(8)$

4

$1(0.8)$

Comorbidities COPD

Cardiovascular disease

Anticoagulant treatment

$19(15.2)$

Antiplatelet treatment

$32(25.6)$

Cancer characteristics

Subtype

Squamous cell carcinoma

$65(52)$

Adenocarcinoma

$31(24.8)$

Other

Cancer stage ${ }^{\#}$

I-IIIA

$37(29.6)$

IIIB-IV

$87(69.6)$

$26(20.8)$

Necrosis or cavitation

91 (72.8)

Anticancer treatments prior to current hospital stay

None

$73(58.4)$

Surgery

Chest radiation therapy

$13(10.4)$

Chemotherapy

$47(37.6)$

Cancer status

Controlled

$11(8.8)$

Progressive

$31(24.8)$

Unknown

$83(66.4)$

Haemoptysis characteristics

Cumulative volume at admission ${ }^{+} \mathrm{mL}$

Haemoglobin $\mathrm{g} \cdot \mathrm{dL}^{-1}$

$200(100-300)$

Main therapeutics in ICU

Mechanical ventilation

$10.6(9.1-12)$

Vasopressors

$38(30.4)$

Transfusion

$21(16.8)$

$29(23.2)$

Haemoptysis therapeutics

Medical treatment

$20(16)$

Bronchial arteriography

$102(81.6)$

Embolisation

$89(71.2)$

Surgery

$18(14.4)$

Survival

ICU

104 (83.2)

Hospital

$86(68.8)$

1 year ${ }^{\S}$

$38(30.4)$

Cancer treatment after ICU discharge

60 (48)

Data are presented as median (interquartile range) or $\mathrm{n}(\%) . \mathrm{N}=125$. ICU: intensive care unit; COPD: chronic obstructive pulmonary disease. ${ }^{\#}$ : in one stage III nonsmall cell lung cancer patient the A or B staging could not be determined; ${ }^{\uparrow}:$ in three patients the location could not be determined; ${ }^{+}:$in 14 patients requiring mechanical ventilation the amount of bleeding could be not evaluated; ${ }^{\S}$ : missing data for two patients.

\section{Discussion}

This study better defined the clinical characteristics and outcome of NSCLC-related severe haemoptysis patients. SCC was the most prevalent cancer causing severe haemoptysis, and central location and advanced stage were the most commonly seen cancer features, while cavitation/necrosis was observed in a quarter of patients. Bronchial artery involvement appeared to be the main underlying bleeding mechanism, with bleeding cessation achieved in $80 \%$ of patients undergoing interventional radiology. In-hospital and 1-year 
TABLE 2 Univariate and multivariate analyses of variables associated with in-hospital mortality

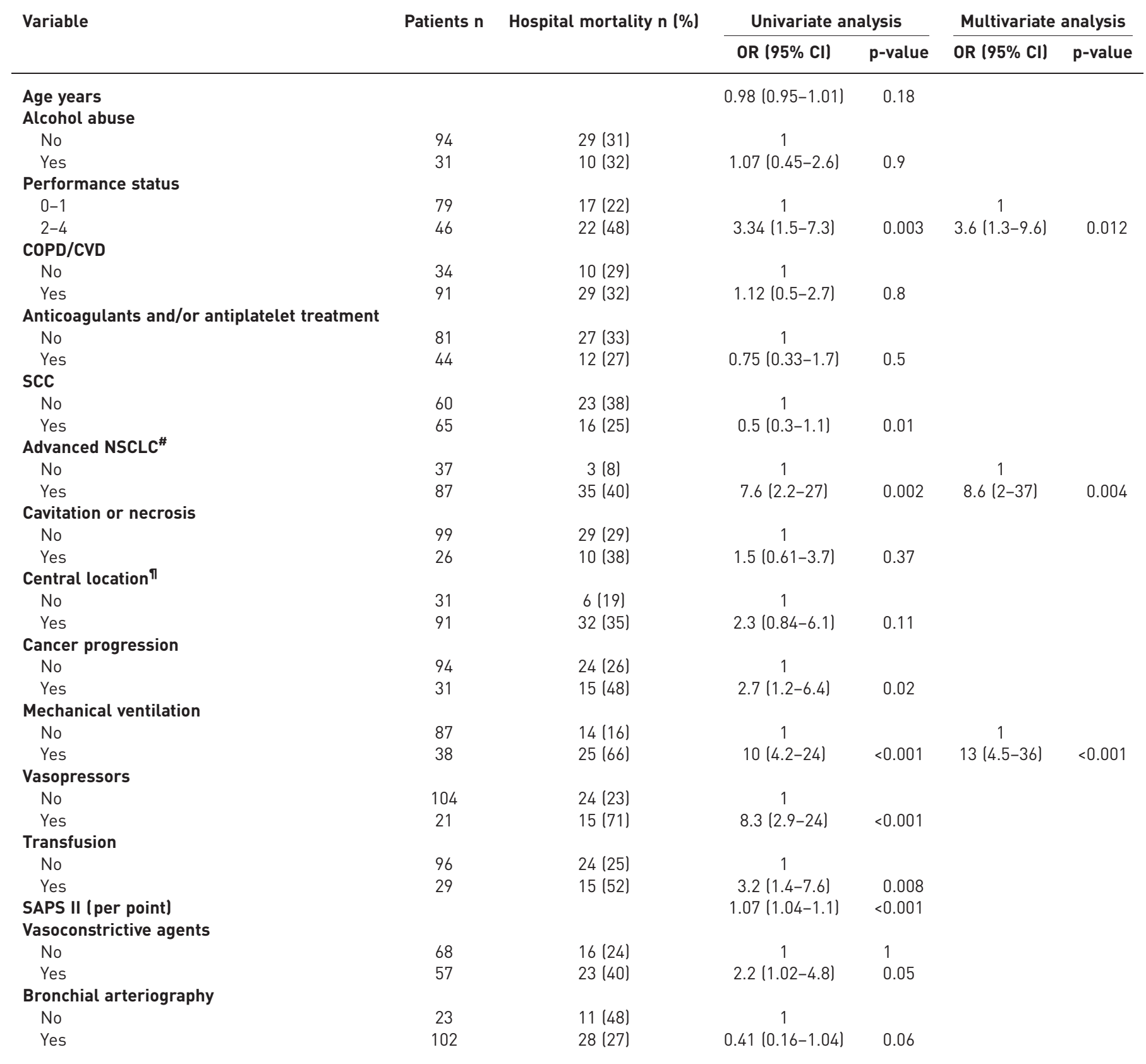

COPD: chronic obstructive pulmonary disease; CVD: cardiovascular disease; SCC: squamous cell carcinoma; NSCLC: nonsmall cell lung cancer; SAPS: Simplified Acute Physiology Score. " : in one stage III NSCLC patient the A or B staging could be not determined; " : central location could not be determined in three patients.

survival rates were $69 \%$ and $30 \%$, respectively. Given these encouraging results, NSCLC-related severe haemoptysis should not be considered so dismissively.

In our study, NSCLC-related haemoptysis accounted for up to 11\% (125 out of 1165 cases) of overall haemoptysis ICU admissions, in contrast with two similar, recently published studies that reported only four NSCLC-related haemoptysis cases $[18,19]$. Yet severe haemoptysis rarely appears in published patient cohorts involving ICU-admitted NSCLC patients $[10,20]$. This may be explained by the restrictive ICU policy of limiting admission of haemoptysis-affected NSCLC patients. Both oncologists and emergency physicians are often unwilling to refer these patients to ICU, on account of their locally advanced/metastatic 
TABLE 3 Univariate and multivariate analyses of variables associated with 1-year survival

\begin{tabular}{|c|c|c|c|c|c|c|}
\hline \multirow[t]{2}{*}{ Variables } & \multirow[t]{2}{*}{ Patients $\mathrm{n}$} & \multirow{2}{*}{$\begin{array}{l}\text { 1-year estimated } \\
\text { survival } \%\end{array}$} & \multicolumn{2}{|c|}{ Univariate analysis } & \multicolumn{2}{|c|}{ Multivariate analysis } \\
\hline & & & $\operatorname{HR}(95 \% \mathrm{CI})$ & p-value & HR $(95 \% \mathrm{CI})$ & $\overline{p \text {-value }}$ \\
\hline Age years & & & $0.99(0.97-1.01)$ & 0.37 & & \\
\hline No & 94 & 35 & 1 & & & \\
\hline Yes & 31 & 20 & $1.41(0.88-2.3)$ & 0.15 & & \\
\hline \multicolumn{7}{|c|}{ Performance status } \\
\hline \multicolumn{7}{|l|}{ COPD/CVD } \\
\hline No & 34 & 40 & 1 & & & \\
\hline Yes & 91 & 28 & $1.3(0.81-2.2)$ & 0.24 & & \\
\hline \multicolumn{7}{|c|}{$\begin{array}{l}\text { Anticoagulants and/or antiplatelet } \\
\text { treatment }\end{array}$} \\
\hline No & 81 & 25 & 1 & & & \\
\hline \multicolumn{7}{|c|}{ Advanced NSCLC\# } \\
\hline No & 37 & 68 & 1 & & 1 & \\
\hline Yes & 87 & 15 & 5. $1(2.8-9.6)$ & $<0.001$ & $4(2.1-7.7)$ & $<0.001$ \\
\hline \multicolumn{7}{|c|}{ Cavitation or necrosis } \\
\hline No & 99 & 37 & 1 & & 1 & \\
\hline Yes & 26 & 7 & $2.01(1.24-3.2)$ & 0.004 & $1.7(1.21-3.2)$ & 0.007 \\
\hline \multicolumn{7}{|c|}{ Central location" } \\
\hline No & 31 & 52 & 1 & & & \\
\hline Yes & 91 & 22 & $2.1(1.2-3.6)$ & 0.011 & & \\
\hline \multicolumn{7}{|c|}{ Cancer progression } \\
\hline No & 94 & 36 & 1 & & 1 & \\
\hline Yes & 31 & 6 & $2.8(1.7-4.4)$ & $<0.001$ & $2(1.01-2.7)$ & 0.04 \\
\hline \multicolumn{7}{|c|}{ Mechanical ventilation } \\
\hline SAPS II I pe & & & $1.04(1.03-1.05)$ & $<0.001$ & & \\
\hline \multicolumn{7}{|c|}{ Vasoconstrictive agents } \\
\hline No & 68 & 37 & 1 & & & \\
\hline Yes & 57 & 23 & $1.5(0.97-2.3)$ & 0.07 & & \\
\hline \multicolumn{7}{|c|}{ Bronchial arteriography } \\
\hline No & 23 & 17 & 1 & & & \\
\hline Yes & 102 & 34 & $0.59(0.35-0.98)$ & 0.04 & & \\
\hline
\end{tabular}

COPD: chronic obstructive pulmonary disease; CVD: cardiovascular disease; SCC: squamous cell carcinoma; NSCLC: nonsmall cell lung cancer; SAPS: Simplified Acute Physiology Score. ": in one stage III NSCLC patient the A or B staging could not be determined; ": central location could not be determined in three patients.

disease, in line with our study $[4,7,21]$, as chemotherapy is unlikely to prove rapidly effective in this setting, thereby aggravating their reluctance.

Previous reports focused on patients with fatal haemoptysis (i.e. SCC, central location and tumour cavitation/necrosis) or with risk of haemoptysis due to bevacizumab therapy (i.e. cavitation) have been confirmed by our data $[6,7,13]$. In our study, central location appeared to be a risk factor for severe haemoptysis. As predicted in a population with advanced SCC [22], a central location was observed in approximately three-quarters of our patients. Considering our adenocarcinoma population, central location (23 (74\%) out of 31 ) was more common than that reported in bevacizumab-treated adenocarcinoma 
patients (1\%) [22]. These different location rates may be accounted for by treatment guidelines that recommend excluding patients with centrally-located adenocarcinoma. Yet the risk of developing haemoptysis for this patient subgroup remains a matter of debate $[6,13]$. Based on a 2004 phase II trial, JoHNSON et al. [5] reported severe haemoptysis to be associated with SCC and tumour necrosis/cavitation, as well as disease located close to major blood vessels. A subsequent phase III trial excluded patients with SCC-related haemoptysis, with a resulting lower incidence of severe haemoptysis (1.9\%) [23]. DANsIN et al. [22] recently found similar incidence rates of severe haemoptysis for central versus non-central location $(0.7 \%$ versus $0.7 \%)$ in bevacizumab patients.

Tumor cavitation was shown to be a risk factor for severe haemoptysis, in particular if cavitation occurred during cancer treatment $[5-7,22]$. Cavitated tumours were observed in 26 (21\%) of our patients, in line with previously reported rates ranging from $10 \%$ (baseline) to $20 \%$ (cancer treatment) [24, 25]. In our series, only two patients suffering from adenocarcinoma exhibited cavitated tumours [25]. In view of the reported severe haemoptysis rates under anti-angiogenic therapy (0.7-2\%), and as half of our patients exhibited SCC, it was not unexpected that none of our patients had undergone anti-angiogenic therapy $[13,22]$. Consequently, we cannot draw any conclusions regarding the contribution of these agents to the haemoptysis occurrence, although a better understanding of the underlying mechanism appears warranted given the benefits of anti-angiogenic therapy in NSCLC [23].

The exact cause or mechanism of haemoptysis should always be sought first, via MDCTA, in order to promptly initiate the most appropriate treatment [15]. In line with previous study data, the main sources of bleeding were bronchial and non-bronchial systemic arteries $(n=104 ; 83 \%)$, with pulmonary arteries involved in only a few cases $[6,15]$. Interventional radiology is currently considered the first-line treatment for severe haemoptysis, although other modalities like surgery and bronchoscopic techniques may be envisaged [26]. As it is associated with high morbidity and mortality, surgery should be restricted to a minority of patients, such as in the event of BAE failure [27]. When experts in bronchoscopic management are available, bronchoscopic therapies may be used to control immediate bleeding $[26,28]$. However, long-term outcome data on these procedures is scarce, and further studies are warranted. In our cohort, BAE was carried out in $102(82 \%)$ patients, with bleeding cessation achieved in $80 \%$, in line with published control rates ranging from 58 to $89 \%$ in NSCLC patients $[2,4,21,29]$. Consequently, BAE has been proven a safe and effective treatment.

ICU admission for patients with non-resectable NSCLC has been well-established [8, 30, 31]. Nonetheless, as patients with severe NSCLC-related haemoptysis were excluded from most studies, the benefits of properly managing these patients have yet to been addressed. In our series, the observed in-hospital and 1-year survival rates of $69 \%$ and $30 \%$, respectively, were consistent with recent reports evaluating BAE in severe haemoptysis $[2,4,21]$. BAE should, thus, be considered whenever possible. According to our findings, vascular interventional radiology might be useful and even life-saving in selected NSCLC-related haemoptysis cases.

Due to the treatment costs, being able to clearly identify patients likely to benefit from this therapeutic option appears crucial. In our study, mechanical ventilation requirement, poor PS and advanced-stage NSCLC proved to be strong predictors of in-hospital mortality, in line with previous reports [8], while 1-year survival was affected by poor PS, in addition to cancer characteristics, such as advanced stage, progression and cavitation/necrosis. These variables should facilitate the decision-making process in favour of "aggressive" haemoptysis management including vascular interventional radiology.

Our study has certain limitations. First, its retrospective design, along with the inclusion of a single referral centre for both haemoptysis and lung cancer cases. By contrast, the strengths of our study include the well-defined inclusion criteria, along with a large series of NSCLC-related haemoptysis cases with proven histological diagnosis. Secondly, although the study covered a $>15$-year period, no difference in terms of haemoptysis severity has been noted and the standard of performing BAE within the first $24 \mathrm{~h}$ of referral has remained unchanged (data not shown). Finally, although precise data on the triage process was not available, patients were selected mainly based on their PS rather than their cancer status. In exceptional cases, bedridden patients (PS 3-4) were admitted, and PS 0-2 patients were primarily admitted in an effort to control their haemoptysis, which is a frightening symptom, whilst their cancer was in progression. The availability of 1-year post-hospital discharge data for all but two patients rendered our management and outcome analyses even more compelling.

In conclusion, our data supports providing appropriate management in the event of NSCLC-related severe haemoptysis. The exact mechanism of severe haemoptysis should be clearly assessed via MDCTA before declaring the patient non-eligible for interventional radiology. ACCP guidelines should be viewed cautiously with respect to BAE, which should be proposed to selected severe haemoptysis patients, as this procedure was proven effective and safe, allowing anticancer therapy to be pursued. Further studies are needed to clarify the impact of anti-angiogenic therapy on haemoptysis incidence, given the benefits of these agents on survival in advanced cancer patients. 


\section{Acknowledgements}

The authors would like to thank Ferima Sanogo and Tabassome Simon (Unité de Recherche Clinique de l'Est Parisien, Hôpital Saint Antoine, Assistance Publique Hôpitaux de Paris, and Université Pierre et Marie Curie, Paris VI, France). The authors wish also to thank Gabrielle Cremer for the revision of the English language.

\section{References}

1 Corey R, Hla KM. Major and massive hemoptysis: reassessment of conservative management. Am J Med Sci 1987; 294: 301-309.

2 Park HS, Kim YI, Kim HY, et al. Bronchial artery and systemic artery embolization in the management of primary lung cancer patients with hemoptysis. Cardiovasc Intervent Radiol 2007; 30: 638-643.

3 Simoff MJ, Lally B, Slade MG, et al. Symptom management in patients with lung cancer: diagnosis and management of lung cancer, 3rd ed: American College of Chest Physicians evidence-based clinical practice guidelines. Chest 2013; 143: Suppl. 5, e455S-e497S.

4 Witt C, Schmidt B, Geisler A, et al. Value of bronchial artery embolisation with platinum coils in tumorous pulmonary bleeding. Eur J Cancer 2000; 36: 1949-1954.

5 Johnson DH, Fehrenbacher L, Novotny WF, et al. Randomized phase II trial comparing bevacizumab plus carboplatin and paclitaxel with carboplatin and paclitaxel alone in previously untreated locally advanced or metastatic non-small-cell lung cancer. J Clin Oncol 2004; 22: 2184-2191.

6 Miller RR, McGregor DH. Hemorrhage from carcinoma of the lung. Cancer 1980; 46: 200-205.

7 Panos RJ, Barr LF, Walsh TJ, et al. Factors associated with fatal hemoptysis in cancer patients. Chest 1988; 94 : $1008-1013$.

8 Soubani AO, Ruckdeschel JC. The outcome of medical intensive care for lung cancer patients: the case for optimism. J Thorac Oncol 2011; 6: 633-638.

9 Oken MM, Creech RH, Tormey DC, et al. Toxicity and response criteria of the Eastern Cooperative Oncology Group. Am J Clin Oncol 1982; 5: 649-655.

10 Soares M, Darmon M, Salluh JI, et al. Prognosis of lung cancer patients with life-threatening complications. Chest 2007; 131: 840-846.

11 Mountain CF. Revisions in the International System for Staging Lung Cancer. Chest 1997; 111: 1710-1717.

12 Chaudhuri MR. Primary pulmonary cavitating carcinomas. Thorax 1973; 28: 354-366.

13 Sandler AB, Schiller JH, Gray R, et al. Retrospective evaluation of the clinical and radiographic risk factors associated with severe pulmonary hemorrhage in first-line advanced, unresectable non-small-cell lung cancer treated with carboplatin and paclitaxel plus bevacizumab. J Clin Oncol 2009; 27: 1405-1412.

14 Le Gall JR, Lemeshow S, Saulnier F. A new Simplified Acute Physiology Score (SAPS II) based on a European/ North American multicenter study. JAMA 1993; 270: 2957-2963.

15 Khalil A, Parrot A, Nedelcu C, et al. Severe hemoptysis of pulmonary arterial origin: signs and role of multidetector row CT angiography. Chest 2008; 133: 212-219.

16 Peduzzi P, Concato J, Kemper E, et al. A simulation study of the number of events per variable in logistic regression analysis. J Clin Epidemiol 1996; 49: 1373-1379.

17 Fartoukh M, Khalil A, Louis L, et al. An integrated approach to diagnosis and management of severe haemoptysis in patients admitted to the intensive care unit: a case series from a referral centre. Respir Res 2007; 8: 11.

18 Mal H, Rullon I, Mellot F, et al. Immediate and long-term results of bronchial artery embolization for life-threatening hemoptysis. Chest 1999; 115: 996-1001.

19 Ong TH, Eng P. Massive hemoptysis requiring intensive care. Intensive Care Med 2003; 29: 317-320.

20 Adam AK, Soubani AO. Outcome and prognostic factors of lung cancer patients admitted to the medical intensive care unit. Eur Respir J 2008; 31: 47-53.

21 Wang GR, Ensor JE, Gupta S, et al. Bronchial artery embolization for the management of hemoptysis in oncology patients: utility and prognostic factors. J Vasc Interv Radiol 2009; 20: 722-729.

22 Dansin E, Cinieri S, Garrido P, et al. MO19390 (SAiL): bleeding events in a phase IV study of first-line bevacizumab with chemotherapy in patients with advanced non-squamous NSCLC. Lung Cancer 2012; 76: 373-379.

23 Sandler A, Gray R, Perry MC, et al. Paclitaxel-carboplatin alone or with bevacizumab for non-small-cell lung cancer. N Engl J Med 2006; 355: 2542-2550.

24 Crabb SJ, Patsios D, Sauerbrei E, et al. Tumor cavitation: impact on objective response evaluation in trials of angiogenesis inhibitors in non-small-cell lung cancer. J Clin Oncol 2009; 27: 404-410.

25 Phernambucq EC, Hartemink KJ, Smit EF, et al. Tumor cavitation in patients with stage III non-small-cell lung cancer undergoing concurrent chemoradiotherapy: incidence and outcomes. J Thorac Oncol 2012; 7: 1271-1275.

26 Sakr L, Dutau H. Massive hemoptysis: an update on the role of bronchoscopy in diagnosis and management. Respiration 2010; 80: 38-58.

27 Andréjak C, Parrot A, Bazelly B, et al. Surgical lung resection for severe hemoptysis. Ann Thorac Surg 2009; 88: 1556-1565.

28 Valipour A, Kreuzer A, Koller H, et al. Bronchoscopy-guided topical hemostatic tamponade therapy for the management of life-threatening hemoptysis. Chest 2005; 127: 2113-2118.

29 Hayakawa K, Tanaka F, Torizuka T, et al. Bronchial artery embolization for hemoptysis: immediate and long-term results. Cardiovasc Intervent Radiol 1992; 15: 154-158.

30 Andréjak C, Terzi N, Thielen S, et al. Admission of advanced lung cancer patients to intensive care unit: a retrospective study of 76 patients. BMC Cancer 2011; 11: 159.

31 Slatore CG, Cecere LM, Letourneau JL, et al. Intensive care unit outcomes among patients with lung cancer in the surveillance, epidemiology, and end results-medicare registry. J Clin Oncol 2012; 30: 1686-1691. 